\title{
Towards a demystification of quantum mechanics
}

\section{Giovanni Organtini*}

Dip. di Fisica Sapienza Università di Roma \& INFN-Sez. di Roma

E-mail: Giovanni.organtiniduniroma1.it

Quantum Mechanics is being included in numerous school programmes as part of the curriculum. Most modern textbooks introduce it emphasising its strange or, at least uncommon, somewhat paradoxical, character. We argue that such an approach is wrong because rather than attracting the interest of students toward the topic, it makes quantum mechanics almost unbelievable and artificial. The resulting perception is that quantum mechanics is in fact not understood at all and that we need a new theory that eventually will supersede it.

In contrast, we propose a new approach on classical physics that, stressing the role of the measurements in physics, introduces the concept of state very early in the curriculum. Such a concept is reviewed on each classical physics topic and the concept of force as a vector is almost abandoned for the concept of interaction defined as something that change the state.

In this way it is possible to introduce quantum mechanics without violating any conviction the students acquired learning classical physics. In other words quantum mechanics appears to be as natural as classical physics, at least from the point of view of the results of the experiments.

EPS-HEP 2017 - Venezia, Italy 5 - 12 July 2017

\footnotetext{
* Speaker.
} 


\section{Introduction}

Physics textbooks [ [ $]^{1}$ often introduce quantum mechanics $(\mathrm{QM})$ as a topic significantly different from classical physics. It is often said that classical physics is much different from QM because of the non conventional nature of quantum phenomena and it is believed that this fact actually makes QM difficult to understand. We argue that the difficulties can be overcome by a suitable introduction of classical physics, as briefly shown below.

As a matter of fact, there are several aspects in which classical physics is not so different from QM, despite what is commonly believed. In particular, the vast majority of classical physics textbooks, introduce the concept of state, however they do not emphasise it enough, while often they neither define it. On the other hand, the concept of state is of capital importance in QM: having a clear idea of what the state represents, helps a lot in the comprehension of QM.

\section{About physics, state and forces}

Physics is often defined as the science dealing with natural phenomena. However, love, pain, dreams, etc., are of no (professional) interest for a physicist, though they are as natural as a thunder or tides. On the other hand, some physicists work on non-natural phenomena like the behaviour of prices on the stock market. A first step toward the comprehension of QM consists in making clear that physics is about measurable quantities. In too many textbooks, only the first chapter is devoted to the discussion of the role of the measurements. Discussing the implications of the role of the measurements in the definition of concepts or tools like vectors, fields, heat, etc., is then desirable to make it clear that any physics concept has an experimental ground. A truly experimental approach to physics already helps in introducing the second disrupting modern physics theory: special relativity.

As a matter of fact, in classical physics the process of measurement is only marginally linked with the concepts developed in it. Physics quantities exist independently from the possibility to be measured. As a very well known example, space and time were for centuries believed to be evident enough that no definition was needed for them. Einstein showed that in fact this is not true. What matters is, in fact, what we measure: not what we believe exists independently from a measurement device. Assuming that time exists irrespective of a clock, makes it impossible to understand time dilation. On the contrary, defining time as the quantity measured by a clock, such a conclusion is rather natural and inevitable. While it is true that, as said, classical physics can be formulated without introducing the measurement problem, its introduction helps in shaping a vision according to which all physically meaningful quantities must be measurable and this, in turn, makes modern physics arguments stronger and more convincing.

A second crucial ingredient for a better understanding of QM, is an early discussion of the concept of state, deeply connected with the possibility to make a measurement of a given quantity: a not so crucial concept in classical physics, but a very important one in QM. The term state is often used in mechanics (the state of a point particle is given when position and velocity are known) and in the theory of gases (the equation of state). However, we did not find any textbook in which the

\footnotetext{
${ }^{1}$ We only mention the two most common in Italian schools, but the situation is quite similar in other countries.
} 
definition of it is discussed. We argue that a correct introduction of this concept and a continuous recall of such a term all along the physics course could be of great benefit in understanding QM.

In mechanics, the state of a point particle is given when both position and velocity are given. We found no textbook in which such a statement is justified, nor a book in which the state is even defined. Often, the state is defined as the collection of data needed to predict te evolution of the system with time, but no discussion follows about the reason for that. In fact, the state of a point particle is given by those two measurable quantities just because they are the only independent measurable quantities for a point particle. The color, the shape or the temperature of a point particle are not included in its state because they are meaningless for it. The mass of a point particle can, in fact, be included in the state, but as long as the mass is considered to be constant, there is no reason for doing that.

Upon the application of a force, the state of the point particle changes, since the velocity of the particle changes. It must be observed that the position and speed of a point particle depends on our choice of the reference frame. However, we can always find a reference frame in which, if no forces act on the particle, its position is constant and given by $\mathbf{x}=(0,0,0)$. With such a choice, a force acting on a point particle causes a variation in its speed (and, as a consequence, in its position in the given special reference frame).

At least in this very special, privileged, reference frame forces cause the change of the state of a point particle: $\mathbf{a}=\frac{\mathbf{F}}{\mathrm{m}}$.

For gases we can apply similar arguments: for them, the concepts of position and velocity are meaningless. Position and velocity of a gas cannot be defined, simply because there is no way to measure them, even in principle. Pressure, volume, temperature and quantity are the only meaningful quantities that can be measured for a gas ${ }^{2}$. These four quantities are not independent on each other. They are connected through the equation of state by means of the gas constant. Applying forces to the container may result in shrinking it (the forces modify the container's shape, that in turn is again part of the its state, that changes upon the application of that forces); the latter, then, applies forces to the gas such that its volume is reduced and its pressure and temperature change according to the equation of state.

With this respect, forces are defined as those things that change the state of a system. Capacitor's charge state is changed by electrostatic forces, while the direction of a magnet, representing its state, changes upon the application of a magnetic force. It is plenty of examples like that, that can be done throughout a physics course.

\section{Quantum mechanics}

Adopting this point of view has a first advantage: later in the curriculum, students are told that the weak force is responsible for nuclear decays. The vast majority of them does not understand at all why we need to introduce a new force to justify nuclear decays. The intuitive definition of a force is something that pulls or push and there is no pull nor push in a nuclear decay.

The reason for this is that our eyes measure relative positions. The intuitive belief is consistent with the above definition of force: forces change the state of objects, i.e. their relative positions.

\footnotetext{
${ }^{2}$ in fact there are other quantities that can be measured, such as color, that however are not usually part of physics investigation, so they are neglected
} 
In nuclear decays it does not make sense to consider position and velocity of the atoms as part of the state of the system, while the mass, number and type of particles in initial and final states are meaningful, because they can be measured. With the above definition, one can easily identify the need for a force that causes a change in state.

Any attempt to measure the position and the speed of an electron in atoms eventually fail because of the Heisenberg principle. The state of an electron in an atom cannot then be defined by its position and velocity. In order to define its state, we must identify a set of quantities that can be measured for it. Those quantities are energy and angular momentum. It does not matter how we measure them: what is important is that there is a mean to obtain their values, while there is not for electrons' speed. That's why the state of an electron in an atom can only be represented as a collection of numbers: its energy $E$ and its angular momentum $J$, together with one of its component $J_{z},\left|E, J, J_{z}\right\rangle$. We then cannot imagine the electron as a planet orbiting around the nucleus: this picture is inconsistent with experiments. What we know is only that the electron is around the nucleus, so we need to imagine it like that: something that surrounds the nucleus, according to Rutherford's experiment. Any force applied to such an electron changes its state. For example, the application of an electromagnetic field can cause it to change its energy or angular momentum state, in a perfect analogy with what happens in classical physics.

Moreover, students tend to consider the Pauli Principle as the result of a sort of interaction between electrons. In fact, with our approach, it can be shown that the Pauli Principle holds for classical systems, too. Two point particles cannot be in the same state given by the collection of position, velocity and mass $m$ : if we even admit that they can occupy the same place and have the same velocity, we can never measure the presence of two particles. At most we can measure the presence of a single particle of mass $2 \mathrm{~m}$. Similarly, two gases cannot be in the same state, since they cannot be in the same container having the same pressure, the same volume and the same temperature they take when separated.

\section{Conclusion}

Quantum mechanics is only apparently surprising when compared to classical physics. In terms of basic concepts, the two paradigms are much more similar than it is commonly believed. Both deals with objects in a state, i.e. a collection of measurable quantities, that changes upon the application of forces, whose role is to change the state of a system. What changes from classical physics is that in quantum mechanics the set of quantities defining the state of a particle is different.

By a coherent and continuous discussion about the concept of state throughout the curriculum, we argue that quantum mechanics can be much better understood and its acceptance can be easier. It is desirable to introduce the above concepts early enough in the curriculum, however we understand the difficulties arising from the unavailability of appropriate textbooks. One can then teach classical physics as in textbooks, provided that crucial topics are reviewed as above prior to start teaching QM.

We introduced this teaching strategy in a class of students in biotechnology. At least qualitatively, the approach was very much appreciated. We look forward to obtain more quantitative results next year. 


\section{References}

[1] U. Amaldi, "Dalla mela di Newton al bosone di Higgs", Zanichelli (2016); C. Romeni, "Fisica e realtà.blu", Zanichelli (2017) 